\section{Response to the Letter \\ to the Editor: \\ Multidisciplinary \\ Considerations \\ in the Implementation \\ of the Findings \\ from the American College \\ of Surgeons Oncology \\ Group (ACOSOG) Z0011 \\ Study: A Practice-Changing \\ Trial}

The letter from Drs. Batsis and Zoras reflects the widespread interest in the results of the ACOSOG Z0011 trial. We agree that thoughtful review of the data is warranted to apply the results in an appropriate population of patients with early-stage breast cancer. As mentioned, one consideration is that the trial did not enroll the 1,900 patients proposed to meet the primary objective. Although it is desirable to achieve the target accrual, several considerations suggest that this would not have altered the outcomes reported. The statistical considerations in the initial design were based on literature anticipating an $80 \%$ 5 year OS rate for patients with one to two positive nodes. However, SLND detects a lower burden of disease than ALND and this, coupled with advances in care, including improvements in imaging, pathology, radiation, and systemic treatment, are likely responsible for the better than anticipated 5 year OS seen in the trial (SLND 92.5\% and ALND 91.8\%). ${ }^{1}$ For ALND to confer an OS benefit, it would need to improve locoregional control. After a median follow-up of 6.3 years, axillary recurrence rates were 0.5 and $0.9 \%$ in the ALND and SLND groups, respectively, ${ }^{2}$ and it is unlikely that additional follow-up would alter these recurrence rates enough to impact OS. Finally, the issue of power is not as relevant in a positive trial, such as Z0011. It was designed as a noninferiority trial with a noninferiority margin of a one-sided hazard ratio of less than 1.3, indicating that SLND alone is not inferior to ALND. The confidence interval (CI) did not cross 1.3 , hence a positive trial. If the trial had been negative and the CI crossed 1.3, there would have been more concern regarding failure to meet target accrual as each event would cause the CI to become narrower and it may have failed to cross 1.3 .

We agree that there is much to learn about the biology of breast cancer and metastasis. Results from NSABP B-04 revealed nodal metastasis in $38 \%$ of the patients undergoing axillary dissection. Only $18 \%$ of patients in the groups without axillary surgery developed clinically detectable nodal disease at follow-up, suggesting that not all tumor cells detected in lymph nodes at surgery become clinically relevant. ${ }^{3}$ Discussion of the Z0011 trial design and results has helped to define a group of patients at low-risk for recurrence in whom ALND may be safely omitted.

The goal of our article was to add our multidisciplinary interpretation of these data. We feel that ALND can be safely omitted in selected women with T1-2, clinically node negative breast cancer who undergo breast conservation therapy with whole breast irradiation and appropriate systemic therapy.

Abigail S. Caudle, MD, Kelly K. Hunt, MD, and Elizabeth A. Mittendorf, MD

Department of Surgical Oncology, University of Texas MD Anderson Cancer Center, Houston, TX

e-mail: khunt@mdanderson.org

Published Online: 2 July 2011

(C) Society of Surgical Oncology 2011

CONFLICTS OF INTEREST None.

\section{REFERENCES}

1. Giuliano A, Hunt K, Ballman K, et al. Axillary dissection vs. no axillary dissection in women with invasive breast cancer and sentinel node metastasis. JAMA. 2011;305:569-75.

2. Giuliano A, McCall L, Beitsch P, et al. Locoregional recurrence after sentinel lymph node dissection with or without axillary dissection in patients with sentinel lymph node metastases: the American College of Surgeons Oncology Group Z0011 randomized trial. Ann Surg. 2010;252:426-32.

3. Fisher B, Bauer M, Margolese R, et al. Five-year results of a randomized clinical trial comparing total mastectomy and segmental mastectomy with or without radiation in the treatment of breast cancer. N Engl J Med. 1985;312:665-73. 